\title{
Beam-test evaluation of the precision timing capabilities of a CMS HGCAL prototype
}

\section{Axel Buchot Perraguin on behalf of the CMS Collaboration}

Laboratoire Leprince-Ringuet,

Ecole Polytechnique, Palaiseau, France

E-mail: axel.buchot.perraguin@cern.ch

The existing CMS endcap calorimeters will be replaced by a High Granularity Calorimeter (HGCAL) for operation at the High-Luminosity (HL) LHC which will start in 2027. Radiation hardness and excellent physics performance will be achieved by utilising silicon pad sensors and SiPM-on-scintillator tiles with high transverse and longitudinal segmentation. One of the major challenges of the HL-LHC will be the high pile-up environment meaning overlapping interactions, with interaction vertices spread in position and time. In order to efficiently reject particles originating from pile-up, precision timing information of the order of $30 \mathrm{ps}$ for a full shower will be of great benefit. In order to meet such performance goals, the HGCAL will provide timing measurements for individual hits with signals above $12 \mathrm{fC}$, equivalent to 3-10 Minimum Ionising Particles (MIPs), such that clusters resulting from particles with $\mathrm{pT}>5 \mathrm{GeV}$ should have a timing resolution better than $30 \mathrm{ps}$.

In order to assess the technical feasibility and physics performance of such a design, beam tests were performed with a prototype of HGCAL silicon modules at the CERN SPS. We present the detector and DAQ components related to the precision timing evaluation, as well as calibration techniques and preliminary results on the timing performance.

The Eighth Annual Conference on Large Hadron Collider Physics - LHCP2020

25-30 May, 2020

Online 


\section{Introduction}

The future phase of the Large Hadron Collider (LHC) is the High Luminosity LHC (HL-LHC) and it is planned for 2027. The HL-LHC aims to accumulate ten times more luminosity that the Phase-I LHC, ending in 2025. This increase of statistics will be useful for precise studies of Higgs boson, Standard Model (SM) and beyond the SM. But, this will lead to five times more pile-up and a higher radiation dose compared to the current dose at LHC. The existing calorimeters won't perfom well enough in such environnement and will be replaced by a High Granularity Calorimeter (HGCAL). It will be an innovative calorimeter with unprecedented granularity (more than six millions channels) providing information on the energy, position and time of the showers.

HGCAL will be the first calorimeter doing fast timing of the order of 30 ps for a full shower and it will be an additional handle to reject particles originating from pile-up. Silicon module prototypes were tested in the SPS beam in order to validate the timing performance [2].

\section{Prototypes and beam test}

Prototype modules are hexagonal boards composed of a silicon sensor divided in 135 channels of hexagonal shape. On the boards, four chips read the signals from 32 cells. These modules are mounted on a copper-tungsten base-plate for the electromagnetic part and on a steel plate for the hadronic one. Particles passing through the material generate a shower (electromagnetic or hadronic), electron-positron pairs generated in the silicon are collected and create an electric signal.

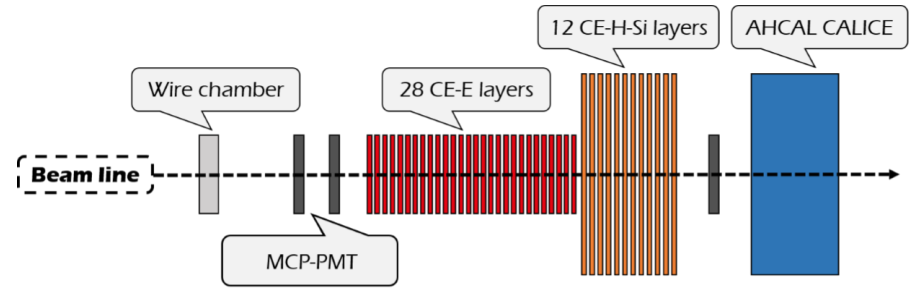

Figure 1: Schematic view of the experimental beam test set up at the CERN SPS, in 2018

In 2018, HGCAL prototype was exposed to beams of electrons, pions and muons with energy varying from 20 to $300 \mathrm{GeV}$, produced at the CERN Super Proton Synchrotron. The experimental set up, shown on Figure 1, is composed of a wire chamber for tracking and position reference, a Micro Channel Plate Photo-Multiplier Tube (MCP-PMT) for timing reference, 28 layers of 1 hexagonal module for the electromagnetic calorimeter (CE-E) and 12 layers of 7 hexagonal modules for the hadronic calorimeter (CE-H-Si). Finally, the setup was completed by the Analog Hadronic Calorimeter (AHCAL) prototype, with scintillators tiles, developped in the context of the CALICE Collaboration [2].

On this study, only electron beams where used, thus just the silicon channels from CE-E modules were taken into account and are read by an Application Specific Integrated Circuit (ASIC) called SKIROC2cms, whose electrical schematic is shown on Figure 2a. In addition to energy information, the quantity of interest regarding timing information is called Time of Arrival (ToA). It is measured from a fast shaper (5 ns). When the trigger is activated after the detected energy reach a 15 MIPs threshold, ToA is measured with a Time to Amplitude Converter (TAC). The voltage 


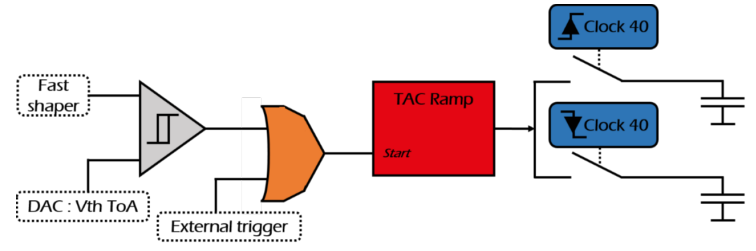

(a) ToA calculation in SKIROC2cms

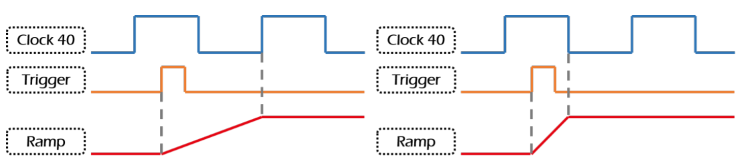

(b) Electrical schematic of ToA calculation

Figure 2: Schematic views of ToA calculation

ramp of the TAC is stopped by clock edge. ToA is measured with respect to two reference points, which are shown on Figure 2b. ToA Rise is obtained when the TAC ramp is stopped at the next rising clock edge and ToA Fall at the next clock falling edge

\section{Calibration}

Before analyzing timing performance, the individual channels need to be calibrated in order to harmonize their time-measurement response. Calibration was done on all the electron beam test data for each single channel. The calibrated time is given by the following formula:

$$
T\left(T o A, E, E_{\text {Layer }}\right)=f_{T o A}\left(\frac{T o A-T o A_{\min }}{T o A_{\max }-T o A_{\min }}\right)+f_{T W}(E)+f_{T W-\text { Layer }}\left(E_{\text {Layer }}\right)
$$

The first term $f_{T o A}$ depends on the normalized ToA and this step of calibration allows to correct for the non-linearity of the ToA distribution due to the ramp saturation in the TAC. Figure 3a shows the non-linear delay depending on the normalised ToA Rise from a single channel. The second term $f_{T W}$ depends on the detected energy of a single channel. In this case the Time Walk (TW) is measured as the difference between the time reference given by an independent source (MCP) and the ToA from the channels. Figure $3 \mathrm{~b}$ shows the difference between ToA Rise from a single channel and MCP, depending on the energy. The last term of the formula $f_{T W \text {-Layer }}$ depends on the total energy of a layer. This calibration step is a layer energy dependent TW calibration. This corrects for an observed layer energy dependant TW. Figure $3 \mathrm{c}$ shows the TW depending on the energy sum of a layer.

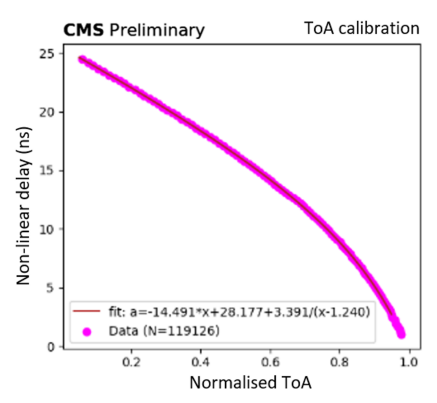

(a) Non-linearity correction

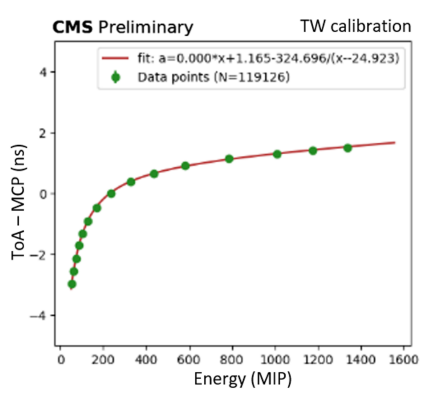

(b) Time Walk correction

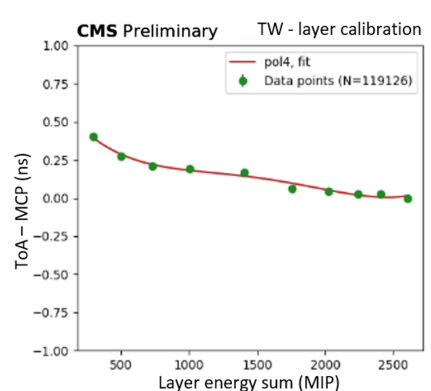

(c) Pedestal shift correction

Figure 3: The three steps of ToA calibration for a single channel 


\section{Timing performances}

For the timing performances, we measure timing performances at different level: the single channel timing resolution, which corresponds to the silicon sensor timing resolution and the full shower resolution, which corresponds to the timing resolution of the shower generated by electrons from beams. In the case of the single cell timing resolution, it was measured on data. It has a stochastic term of 10-20 nsMIP and a constant term around 50 ps [3]. For the full shower timing resolution, the analysis was currently only done for Monte Carlo simulation.

Before getting the resolution depending on the particle energy, the shower timing determination is obtained thanks to the following steps. First, a per channel combination of ToA Rise \& Fall is done, half of the clock period (12.5 ns) are added to ToA Fall to match ToA Rise. Next, an outlier removal, which keep only timing values within the shortest $68 \%$ interval of timing values per event, is done in order to improve the timing resolution. And finally, an energy weighting is done for each event with $t_{\text {weighted }}=\frac{\sum t_{i} E_{i}}{\sum E_{i}}$ where $\mathrm{i}$ is cell indice.

After these steps applied, ToA distributions have gaussian shape and timing resolution is extracted from gaussian fits to the combined time distributions. The Figure $4 \mathrm{a}$ is the calibrated ToA distribution for $100 \mathrm{GeV}$ electron beams.

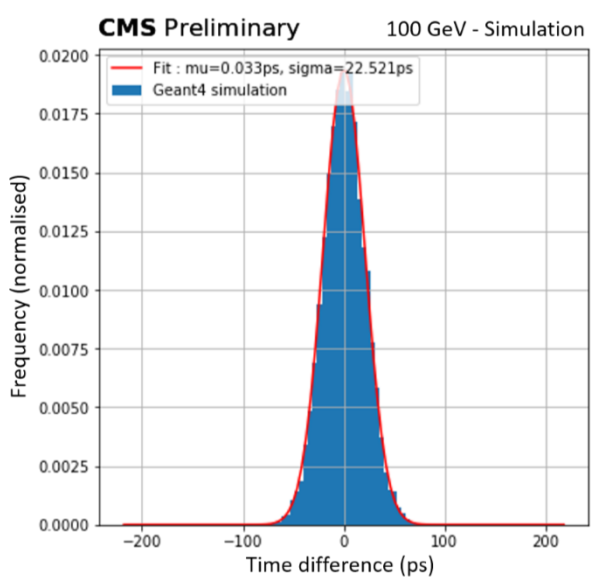

(a) Calibrated Time of Arrival distribution

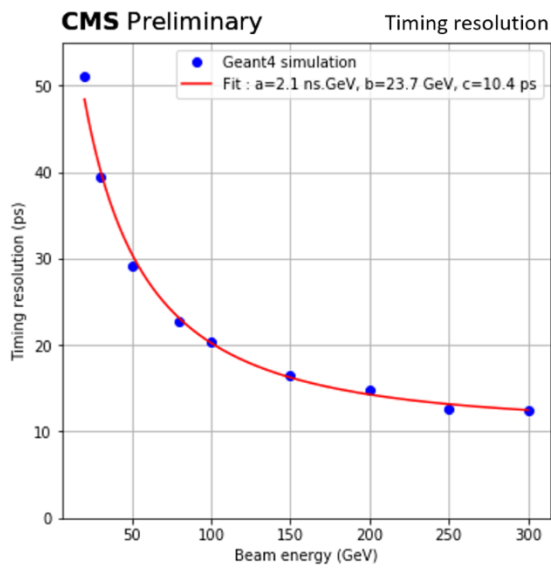

(b) Timing resolution depending on the energy

Figure 4: Calibrated ToA distribution (100 GeV electrons) \& shower timing resolution in CE-E

Timing resolution, represented on Figure $4 \mathrm{~b}$ was done for $28 \mathrm{CE}-\mathrm{E}$ layers and electron beams. It was obtained as the standard deviation from the gaussian fits. Points were fitted with: $y=\frac{a}{x-b} \oplus c$. It has a stochastic term of $2.1 \mathrm{nsGeV}$ and a constant term of $10.4 \mathrm{ps}$ which satisfies the specifications.

\section{Conclusion}

Beam tests were performed at CERN of HGCAL prototypes silicon modules and time calibration was done for the ToA measurement of the prototype ASIC. Full shower resolution from the Geant 4 simulation has a 10.4 ps constant term and was achieved with assumption of ideal calibration and absence of systematic effects. But single channel constant term from data is around $50 \mathrm{ps.}$ Further understanding of systematic effects have to be carried out. 


\section{References}

[1] CMS Collaboration, "The Phase-2 Upgrade of the CMS endcap calorimeter," Technical Design Report, CERN-LHCC-2017-023, CMS-TDR-17-007

[2] F. Sefkow \& F. Simon [CALICE Collaboration], (2019)," A highly granular SiPM-on-tile calorimeter prototype". Journal of Physics: Conference Series. 1162. 012012. 10.1088/17426596/1162/1/012012.

[3] A. Lobanov [CMS Collaboration], "Precision timing calorimetry with the CMS HGCAL", 3rd Conference on Calorimetry for the High Energy Frontier, Nov 2019, Fukuoka, Japan 